Supporting Information for

\title{
A Flavo-diiron Protein from Desulfovibrio vulgaris with Oxidase and Nitric Oxide Reductase Activities. Evidence for an In vivo Nitric Oxide Scavenging Function $^{\dagger}$
}

Radu Silaghi-Dumitrescu, Kim Yong Ng, Rathinam Viswanathan, and Donald M. Kurtz Jr.* Contribution from the Department of Chemistry and Center for Metalloenzyme Studies, University of Georgia, Athens, GA 30602 USA

${ }^{\dagger}$ This work was supported by NIH Grant GM40388 (D.M.K).

*To whom correspondence should be addressed at the Department of Chemistry. Phone: 706542-2016. Fax: 706-542-9454. E-mail: kurtz@chem.uga.edu. 
1

Mt MSQPVAITDGIYWVGAVDWNIRYFHGPAFSTHRGTTYNAYLIV-DDKTALVDTVYEPFKE

DV 1 ---MRKIVESVYWVGAVDWDRRLFDSLVPLPD-GTTYNAYLVEGSEKTALIDAVDPDMVD

DV2 -MHPIEIKKDIFWVGVVDHNSRDFHGYSLSPQ-GTTYNAYVVK-DEKTVLFDTVKHDFTD

Dg -MQATKIIDGFHLVGAIDWNSRDFHGYTLSPM-GTTYNAYLVE-DEKTTLFDTVKAEYKG

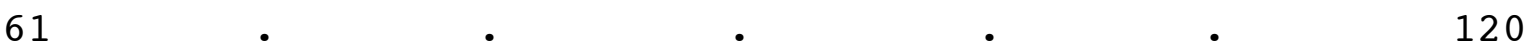

Mt ELIAKLKQIKDPVKLDYLVVNHTESDHAGAFPAIMELCPDAHVLCTQRAFDSLKAHYSHI

DV1 TLLGHLDGVE---KLDYVISQHAEQDHSGTIALVLDLYPEAKVVTNAKAKSMLMDLLLIP

DV2 TMLCRLSRVVEPCKIDYIVCNHLEPDHAGALPELIARCKPEKIFCSPMGLKAMEAHFDTT

Dg ELLCGIASVIDPKKIDYLVIQHLELDHAGALPALIEACQPEKIFTSSLGQKAMESHFHYK

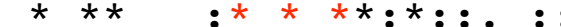

Mt DFNYTIVKTGTSVSLGKRSLTF IEAPMLHWPDSMFTYVPEEALLLPNDAFGQHIATSVRF

DV1 DDKFIVVGDGETLSLGDKTLTF ILTPWVHWPETMSTYLAEDKILFSCDFFGSHIATSDLF

DV2 GWPVEVVKTGDSISIGKRTIHFVETRMLHWPDSMVSYIPEDKLLICNDAFGQNIASTERY

Dg DWPVQVVKHGETLSLGKRTVTFYETRMLHWPDSMVSWFADEKVLISNDIFGQNIAASERF

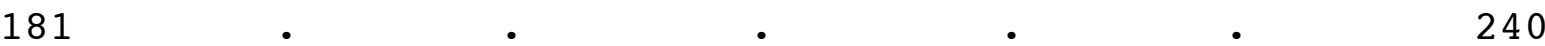

Mt DDQVDAGLIMDEAAKYYANILMPFSNLITKKLDEIQKINLAIKTIAPSHGIIWR--KDPG

DV 1 VRDQGR--VHEAAKRYFGEIMMPFRTIIAKNLEKLG--PYDIRMIAPSHGQIYD---SPG

DV2 ADEIDRSALFHAMKEYYHNIVLPFSPIVLKTLAQIEQLGLDIDMLAPDHGLIFRGYDDVK

Dg SDQIPVHTLERAMREYYANIVNPYAPQTLKAIETLVGAGVAPEF ICPDHGVIFRGADQCT

2412.00300

Mt RIIEAYARWAEGQGKAKAVIAYDTMWLSTEKMAHALMDGLVAGGCEVKLFKLSVSDRNDV

DV1 WIIDAYRDWVSGVAHNLVAFPFVSMHGSTRLMVDHLAAALSERDVRVELFNLAVTDIGKL

DV2 YALDTYRIFAEQKPQKRAVIVYDTMWHSTEKMASAIAEGLESVGVPTRVMWLKANHHSAV

Dg FAVQKYVEYAEQKPTNKVVIFYDSMWHSTEKMARVLAESFRDEGCTVKLMWCKACHHSQI

$301 \quad$. $30 \quad$. 360

Mt IKEILDARAVLVGSPTINNDILPVVSPLLDDLVGLRPKNKVGLAFGAYGWGGGAQKILEE

DV1 AMSLVDAGTIVLGTPTVLAGPHPMAAYAAFLANALRPKAKYLSIVGSYGWGGKTVETLAG

DV2 MTELADCGAVLVGSPTHNNGILPAVAAMLTYMKGLRPQNRIGAAFGSFGWSGESVKSITE

Dg MSEISDAGAVIVGSPTHNNGILPYVAGTLQYIKGLRPQNKIGGAFGSFGWSGESTKVLAE

* $* * * \quad * \quad * * *$ * $*$ *

$361 \quad$ •

Mt RLKAAKIELIAEPGPTVQWVPRGEDLQRCYELGRKI-AARIAD-----

DV1 MIPNLKVEVL-DPVLCKG-LPT-DDTYGALDRLADAIAAKHKESGFQR

DV2 WLQSMGMETPVDP-VKVKHVPTHDTYRQCFEMGQAVGRALIEKCGG--

Dg WLTGMGFDMPATP-VKVKNVPTHADYEQLKTMAQTIARALKAKLAA--

* * *

Figure 1S. ClustalW amino acid sequence alignment of $M$. thermoacetica FprA (Mt, NCBI accession number AAG00802), D. gigas ROO (Dg, AAG34792) and the two D. vulgaris FprA homologs, (Dv1, AAS96489, genome locus ID DVU2013, encoded upstream of hcp; Dv2, YP_012395, DVU3185, encoded downstream of rbo-rub). Stars indicate fully conserved residues. Diiron ligand residues in D. gigas ROO are marked with red stars, and residues involved in a flavin-binding signature are underlined. 